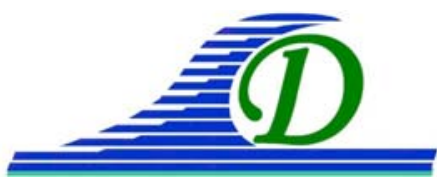
XIII ${ }^{\text {èmes }}$ Journées Nationales Génie Côtier - Génie Civil Dunkerque, 2-4 juillet 2014

DOI:10.5150/jngcgc.2014.006 @ Editions Paralia CFL

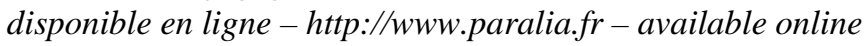

\title{
Projet EMACOP : modélisation numérique des vagues à l'approche de la digue d'Esquibien par le code SWASH
}

\author{
Jonathan COIGNARD ${ }^{1}$, Bertrand MICHARD ${ }^{2}$, \\ Jean-François FILIPOT ${ }^{3}$, Philippe SERGENT ${ }^{2}$
}

1. Université de Technologie de Compiègne, rue Roger Couttolenc, 60200, Compiègne, France. jonathan.coignard@etu.utc.fr

2. CEREMA / DTecEMF, Technopôle Brest-Iroise BP 529280 Plouzané, France. bertrand.michard@cerema.fr, philippe.sergent@cerema.fr

3. France Energies Marines, Technopôle Brest-Iroise 29280 Plouzané, France. jean.francois.filipot@france-energies-marines.org

\section{Résumé :}

Dans un contexte de développement durable, la tendance actuelle est de se tourner vers des sources alternatives de production d'énergie. Le projet National de Recherche EMACOP (Energies MArines COtières et Portuaires) a entre autres pour objectif de définir le potentiel houlomoteur aux abords d'ouvrages côtiers. Dans le présent travail, une simulation numérique des transformations induites aux vagues à l'approche du site d'Esquibien (Finistère) est réalisée. La modélisation numérique des vagues est effectuée avec le code de calcul SWASH (ZIJLEMA et al., 2011). Pour chaque simulation, des résultats d'élévation temporelle, de hauteur significative et de spectre directionnel sont obtenus. Les analyses des simulations portent sur l'étude des conditions hivernales moyennes, mais aussi sur l'influence des directions de provenance des vagues, des hauteurs et périodes des houles et des différents niveaux d'eau pour le site d'Esquibien. Mots-clés : EMACOP, Energies marine, Modélisation numérique, Transformation des vagues, Code SWASH, Site, Esquibien, Potentiel énergétique, Génie côtier.

\section{Introduction}

La transition énergétique engagée en France implique un développement important des énergies marines renouvelables dans les années à venir. A ce titre, un des objectifs du projet national EMACOP (Energies MArines COtières et Portuaires) est de caractériser le potentiel houlomoteur aux abords des ouvrages côtiers (http://www.emacop.fr/).

Un travail d'identification et d'évaluation du potentiel (MICHARD et al., 2013) a été réalisé dans la phase de démarrage, afin de sélectionner les sites les plus pertinents. Pour approfondir le potentiel estimé comme prometteur sur le site d'Esquibien en baie d'Audierne (Finistère), le travail présenté porte sur la simulation numérique des transformations induites aux vagues à l'approche de la côte. 


\section{Thème 1 - Hydrodynamique côtière}

La modélisation numérique des vagues est réalisée avec le code de calcul SWASH v1.2 (ZIJLEMA et al., 2011). Le code SWASH (pour Simulating WAves till Shore) est développé par l'Université de Technologie de Delft (NL) dans la continuité du code SWAN, il résout les équations non linéaires en faible profondeur en y ajoutant un terme de pression non-hydrostatique. Il offre la possibilité d'étudier la propagation des vagues, les zones de déferlements des vagues, l'agitation au sein des ports et le franchissement des ouvrages côtiers de façon déterministe (http://swash.sourceforge.net). Ce code de calcul se veut flexible sur l'échelle des simulations réalisables, de la seconde à l'heure et du mètre aux kilomètres. Il est à souligner que le code SWASH ne correspond pas à un modèle du type Boussinesq, la différence résultant dans la modélisation de la structure verticale au sein de la colonne d'eau, et dans la prise en compte d'un terme de pression non-hydrostatique (ZIJLEMA et al., 2011). Ainsi ce code de modélisation numérique propose un juste compromis entre temps de calcul et précision, cette dernière pouvant être en autre améliorée par une discrétisation verticale plus fine. Le site web du code SWASH renvoie vers des thèses et articles en lien avec le code sur des thématiques variées comme, la transformation des vagues à l'approche d'une plage et l'interaction des vagues avec une digue. De plus, des modélisations test ont été réalisées au préalable en comparaison avec les codes REFONDE (CEREMA) et ARTEMIS (EDF/LNHE), celles-ci montrent une bonne cohérence des résultats obtenus.

L'orientation vers le code de calcul SWASH s'est faite par rapport aux données océanographiques et aux objectifs de l'étude. Dans la mesure où, les premières données d'états de mer disponibles se trouvent à $10,5 \mathrm{~km}$ au sud du site d'Esquibien, et que l'objectif de l'étude est de comprendre l'évolution du potentiel énergétique des vagues aux travers d'évènements caractéristiques (basse mer, mer de vent, ...) in situ. Le code SWASH est alors adapté à la situation, demandant de réaliser des simulations en proche côtier sur une durée de 40min, et préférable à la construction d'un modèle plus large sur une période plus longue apportant des grandeurs moyennées sur la zone d'étude. De plus, le code SWASH a été choisi par rapport aux codes ARTEMIS et REFONDE, notamment pour sa gestion automatique des zones "sèches" et "mouillées", ce qui a rendu plus facile l'étude de différents niveaux d'eau, enfin son caractère novateur a été une motivation supplémentaire.

Concernant les résultats des simulations réalisées sur le site d’Esquibien, ils ont pu être appréciés de façon qualitative par des observations in situ, mais devront être confrontés à la campagne de mesure bouée plus capteurs de pressions lancée en décembre 2013. Les analyses post-simulations permettent de mettre en avant des zones de concentration et de dissipation de l'énergie des vagues, pour des conditions d'états de mer hivernales variées.

La construction du modèle numérique de calcul SWASH, les résultats obtenus et l’analyse de la sensibilité des différents paramètres sont présentés dans cet article. 


\section{XIII ${ }^{\text {èmes }}$ Journées Nationales Génie Côtier - Génie Civil \\ Dunkerque, 2-4 juillet 2014}

\section{Construction du modèle numérique}

\subsection{Modélisation de la bathymétrie}

La bathymétrie a été digitalisée à partir de la carte marine SHOM du port d'Audierne ( $\mathrm{N}^{\circ} 7147$ de la chaussée de Sein à la pointe de Penmarc’h) dans le repère Lambert II étendu, les cotes bathymétriques sont prises par rapport au zéro hydrographique. La bathymétrie interpolée tous les mètres avec le logiciel BlueKenue (Centre Canadien d'hydraulique) a pour origine le point de coordonnées (85 327 ; 2354 915) dans le repère Lambert II étendu. La bathymétrie a été modélisée sur un domaine plus important que la zone de calcul, de façon à pouvoir faire pivoter la zone de simulation librement, notamment pour préserver la propagation des lignes de crête perpendiculaire à la frontière entrante du modèle lorsque celles-ci ont une composante ouest.

\subsection{Paramétrage du modèle}

La taille des mailles horizontales est de $4 \mathrm{~m}$ selon l'axe des $x$ et de $2 \mathrm{~m}$ selon l'axe des $y$ (sur une emprise de $3,5 \mathrm{~km}^{2}$ ). Cette résolution permet d'avoir au moins 50 mailles par longueur d'onde en entrée de modèle, tout en relâchant la largeur des mailles à $4 \mathrm{~m}$ dans la direction opposée à la propagation des vagues pour alléger les calculs.

La colonne d'eau est discrétisée en 2 couches verticales, pour tenir compte des hautes fréquences (maximum de 0,3 Hz), et avoir une bonne représentation de la dispersion des vagues (avec une erreur de $1 \%$ sur la vitesse des vagues).

Le pas de temps de calcul n'est pas homogène pour toutes les simulations. Dans la mesure où certaines simulations induisent des courants plus forts, le pas de temps a dû être baissé, ainsi les pas de temps varient de $0,05 \mathrm{~s}$ à $0,01 \mathrm{~s}$.

Les conditions de forçage initiales sont obtenues en utilisant les 10 premières minutes de la simulation pour atteindre un état agité "initial".

Les conditions aux limites sont modélisées par des vagues dont les lignes de crête sont parallèles à la frontière sud, ainsi que par des absorbeurs positionnés à la bordure nord (largeur fixée à $200 \mathrm{~m}$ ), ouest (largeur fixée à $100 \mathrm{~m}$ ) et est (largeur fixée à $100 \mathrm{~m}$ ), qui ont pour but d'annuler les effets de réflexion aux frontières.

\section{Choix des états de mer simulés}

\subsection{Utilisation des données reconstituées ANEMOC}

Le point 2511 de l'atlas numérique de houle ANEMOC (CEREMA/DTecEMF et EDF/LNHE ; http://anemoc.cetmef.developpement-durable.gouv.fr) a été utilisé pour récupérer des données hindcast sur 23 ans (période de 1979 à 2003). Il se situe à 10,5 $\mathrm{km}$ au sud et 3km à l'est d'Esquibien. Le traitement des données (MICHARD et al., 2013) a permis d'obtenir des statistiques représentatives sur les paramètres de hauteur significative, période et direction des vagues. 


\subsection{Utilisation des cartes de vagues PREVIMER}

A partir de l'analyse des données PREVIMER (IFREMER, http://www.previmer.org) sur la période de septembre à décembre 2013, il a été possible d'estimer la variabilité moyenne de la hauteur et de la direction des vagues, entre le point ANEMOC N 2511 et l'entrée sud du modèle numérique d'Esquibien. Les résultats obtenus montrent que l'angle de provenance des houles varie de $40^{\circ}$ à $50^{\circ}$ et la hauteur significative varie de $0,7 \mathrm{~m}$.

\subsection{Combinaison des paramètres retenus}

Les analyses précédentes permettent de retenir, pour chaque paramètre spectral, une valeur moyenne susceptible d'être rencontrée à la frontière sud du modèle numérique, et 2 autres valeurs qui l'encadrent.

De plus 3 niveaux d'eau correspondant à la basse mer, mi marée et pleine mer sont étudiés pour une marée de vive eau (coefficient de 95, le 6 novembre 2013).

Tableau 1. Les 7 simulations de l'étude de sensibilité réalisées sur le site d'Esquibien.

\begin{tabular}{llllllll}
\hline $\begin{array}{l}\text { Simulations / } \\
\text { Paramètres }\end{array}$ & Référence & $\begin{array}{l}\text { Direction } \\
\mathbf{1}\end{array}$ & $\begin{array}{l}\text { Direction } \\
\mathbf{2}\end{array}$ & Triplet 1 & Triplet 2 & $\begin{array}{l}\text { Niveau } \\
\text { d'eau 1 }\end{array}$ & $\begin{array}{l}\text { Niveau } \\
\text { d'eau 2 }\end{array}$ \\
\hline Direction & $200^{\circ} \mathrm{N}$ & $180^{\circ} \mathrm{N}$ & $220^{\circ} \mathrm{N}$ & $200^{\circ} \mathrm{N}$ & $200^{\circ} \mathrm{N}$ & $200^{\circ} \mathrm{N}$ & $200^{\circ} \mathrm{N}$ \\
Niveau d'eau & $3 \mathrm{~m}$ & $3 \mathrm{~m}$ & $3 \mathrm{~m}$ & $3 \mathrm{~m}$ & $3 \mathrm{~m}$ & $0,8 \mathrm{~m}$ & $5,25 \mathrm{~m}$ \\
Haut. significative & $2 \mathrm{~m}$ & $2 \mathrm{~m}$ & $2 \mathrm{~m}$ & $1 \mathrm{~m}$ & $2 \mathrm{~m}$ & $2 \mathrm{~m}$ & $2 \mathrm{~m}$ \\
Période de pic & $10,3 \mathrm{~s}$ & $10,3 \mathrm{~s}$ & $10,3 \mathrm{~s}$ & $7 \mathrm{~s}$ & $13 \mathrm{~s}$ & $10,3 \mathrm{~s}$ & $10,3 \mathrm{~s}$ \\
Etalement & $25^{\circ}$ & $25^{\circ}$ & $25^{\circ}$ & $37,5^{\circ}$ & $18^{\circ}$ & $25^{\circ}$ & $25^{\circ}$ \\
\hline
\end{tabular}

\section{Résultats des simulations effectuées}

\subsection{Présentation des résultats}

Les résultats obtenus par SWASH ont été traités avec MATLAB et les boites à outils WAFO (www.maths.lth.se/matstat/wafo) et DIWASP (www.metocean.co.nz). Les résultats qui ont été exploités en sortie de modélisation sont les suivants :

- l'élévation de la surface libre à t=40 mn sur l'intégralité du domaine avec MATLAB,

- la hauteur significative pendant une durée de 30 min sur le domaine avec MATLAB,

- les spectres directionnels avec DIWASP (localisés sur la figure 1) et la puissance linéique correspondante calculée à partir de la formule suivante (EPRI, 2011).

$$
P_{l}=\frac{\rho g^{2}}{4 \pi} \int_{0}^{\infty} \frac{S(f)}{f}\left[\left(1+\frac{2 k_{f} d}{\sinh \left(2 k_{f} d\right)}\right) \tanh \left(k_{f} d\right)\right] d f
$$

Le temps de simulation varie de $20 \mathrm{~h}$ à $48 \mathrm{~h}$ selon les simulations sur un ordinateur disposant d’un processeur Intel Xeon CPU E3- 1245 V2 @ 3,40 GHz. 


\section{XIII ${ }^{\text {èmes }}$ Journées Nationales Génie Côtier - Génie Civil \\ Dunkerque, 2-4 juillet 2014}

4.2 Résultats pour la simulation de référence

Les graphiques des figures 2, 3, 4, 5, 6 et 7 représentent la simulation d'un état de mer de référence (colonne 1 du tableau 1). La simulation de référence permet de mettre en avant la dynamique du site face à des conditions hivernales moyennes.

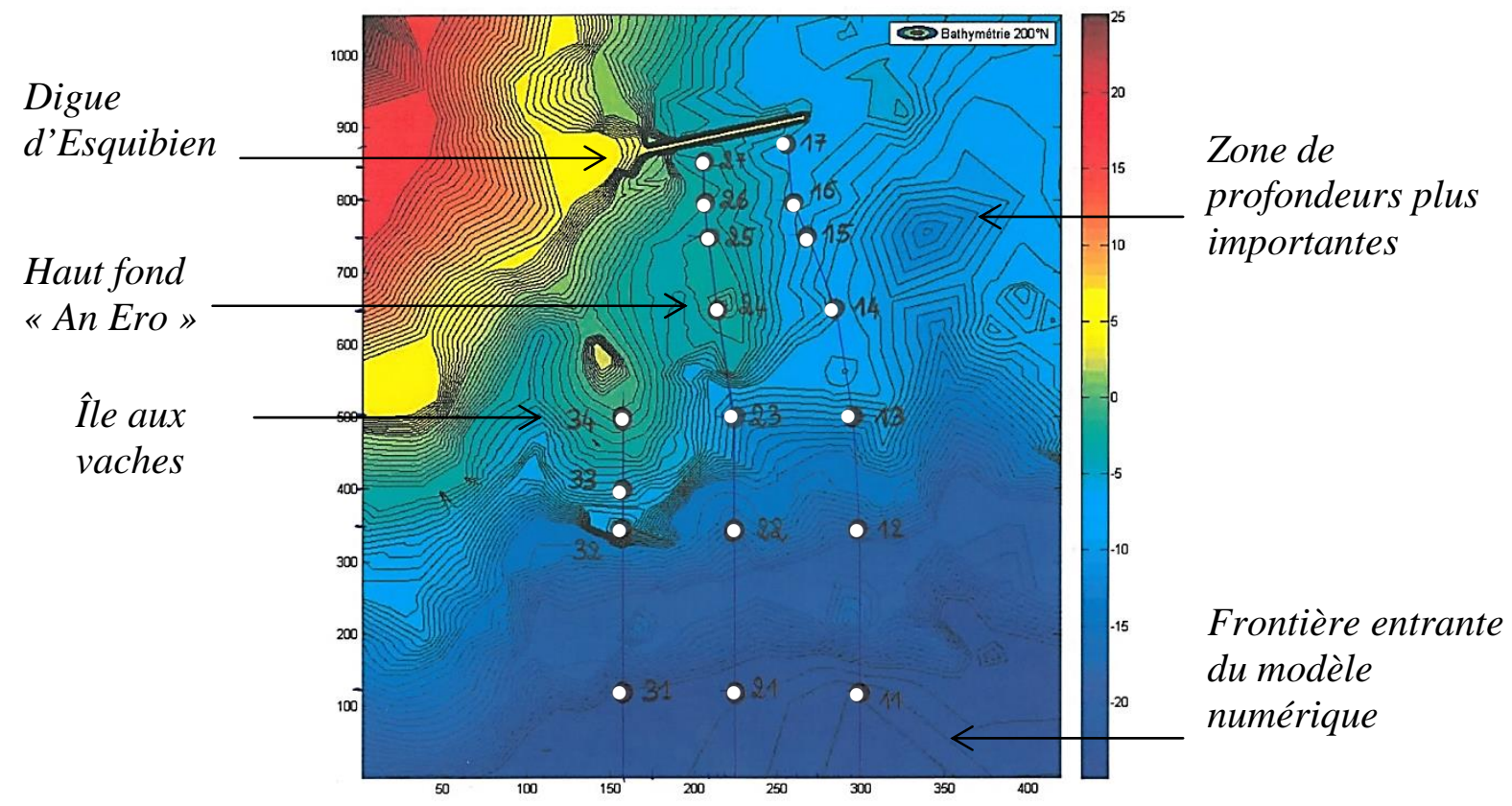

Figure 1. Emplacement des spectres directionnels et représentation bathymétrique graduée en nombre de mailles $2 m(x)$ par $4 m(y)$.

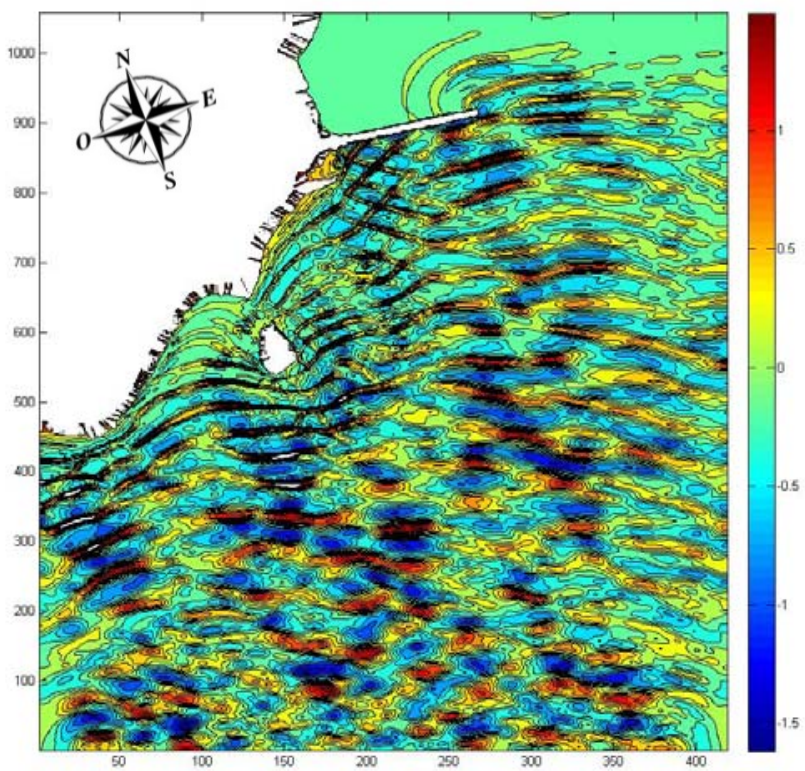

Figure 2. Résultat de l'élévation de la surface libre ( $t=40 \mathrm{~min})$ pour la simulation de référence ( $\mathrm{HmO}=2 \mathrm{~m}, \mathrm{~T}_{p}=10,3 \mathrm{~s}$, Dir $=200^{\circ} \mathrm{N}$, Etal $=25^{\circ}$ Niveau d'eau mi-marée de 3 $m)$. 


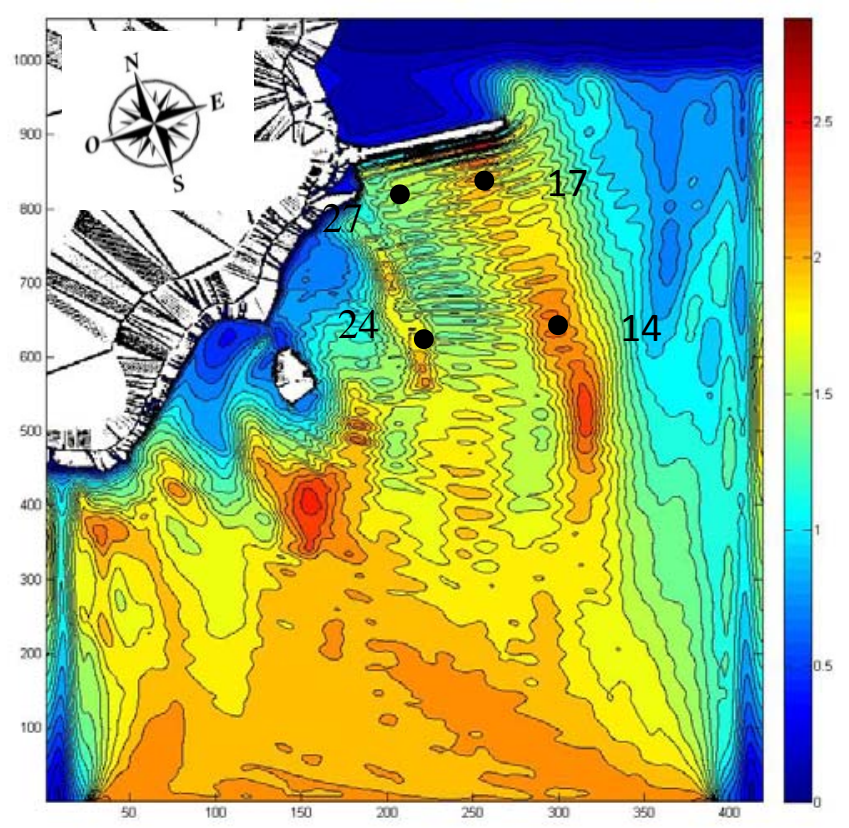

Figure 3. Résultat de la hauteur significative de la houle $\left(H_{s}\right)$ pour la simulation de référence avec la position des spectres "14", "17", "24" et "27".

\section{Discussion des résultats}

\subsection{Analyse de la situation de référence}

L'observation des hauteurs significatives $\left(H_{s}\right)$ montre que les houles de direction de provenance $200^{\circ} \mathrm{N}$ convergent en partie sur l'île aux vaches (localisée sur la figure 1), ce qui a pour effet d'abriter des vagues une partie de la digue. Deux zones principales de shoaling se démarquent, la première en avant de l'île aux vaches, et la deuxième au sud du point "14" (figure 1) avec une augmentation de la hauteur des vagues de 25\% par rapport à la frontière sud.

A l'inverse, les résultats indiquent à l'est de la digue une zone où les valeurs de $H_{s}$ sont plus faibles. En effet, les fonds de $6 \mathrm{~m} \mathrm{CM}$ à l'est remontent rapidement à $3 \mathrm{~m} \mathrm{CM}$ en allant vers l'ouest, ainsi les vagues se réfractent, et s'orientent en direction de la digue $\left(190^{\circ} \mathrm{N}\right)$, mettant en évidence le phénomène de caustique.

A l'extrémité de la digue, et à intervalle régulier d'une demi-longueur d'onde, des variations de $10 \%$ des hauteurs significatives se produisent, dû à la réflexion sur la digue. Le phénomène correspond à une interférence entre l'onde réfléchie et l'onde incidente, créant localement des ventres où les amplitudes sont supérieures. Le spectre "17" (figure 5) montre que l’onde incidente après avoir été réfléchie conserve sa période de $10 \mathrm{~s}$.

Les spectres directionnels le long de la courbe $n^{\circ} 1$ (figure 1 ) montrent que, les vagues changent fortement de direction entre les points "13" et "14", c'est à ce moment qu'elles s’orientent vers la digue. Au point "17" la puissance linéique est élevée $\left(P_{l}=20,4 \mathrm{~kW} / \mathrm{m}\right.$ 


\section{XIII ${ }^{\text {èmes }}$ Journées Nationales Génie Côtier - Génie Civil \\ Dunkerque, 2-4 juillet 2014}

soit 92\% de celle à la limite sud) ce qui s'explique par la prise en compte de l'onde réfléchie. Le point "15" est marqué par l'influence grandissante des interactions non linéaires, qui forment un pic d'énergie pour des périodes deux fois inférieures (de $5 \mathrm{~s}$ ). Le long de la courbe $\mathrm{n}^{\circ} 2$ (figure 1), la direction des vagues varie de $80^{\circ}$ entre les points "23" et "24". La puissance linéique au point "24" (figure 6) est relativement faible (25,5\% de celle au point "21"), avec néanmoins une hauteur significative de 1,6 m.

\subsection{Résultats de l'étude de sensibilité pour les 7 simulations réalisées}

Lorsque les houles ont une composante ouest, la côte capte l'énergie des vagues qui se dissipe sur l'île aux vaches. Au contraire, les houles venant du sud frappent directement la digue. Au point "15" pour la simulation de provenance $180^{\circ} \mathrm{N}$, la puissance linéique est de $P_{l}=15,8 \mathrm{~kW} / \mathrm{m}$, pour la simulation de provenance $200^{\circ} \mathrm{N}, P_{l}=15 \mathrm{~kW} / \mathrm{m}$, enfin pour la simulation $220^{\circ} \mathrm{N}, P_{l}=6,8 \mathrm{~kW} / \mathrm{m}$. La direction de provenance des vagues à $2 \mathrm{~km}$ au large d'Esquibien influe donc de manière significative la répartition de l'énergie à la côte.

L'énergie transportée par les vagues dépend du couple $H_{m 0}, T_{p}$. Une diminution de la hauteur ou de la période engendre des énergies moindres à la côte. Néanmoins, les résultats obtenus montrent que la répartition de l'énergie joue un rôle, ainsi par endroits les différences sont atténuées. Au point "15" la différence de potentiel atteint un facteur 20, alors que au point "24", elle ne dépasse pas un facteur 4, pour les deux spectres testés.

Le niveau d'eau expose plus ou moins certaines parties de la digue. A basse mer, l'île aux vaches et le haut fond "An Ero" (figure 1) jouent des rôles protecteurs. A pleine mer, les vagues réfractent moins avant d'arriver sur la digue, de plus, certaines zones ne font plus déferler les vagues, et deviennent des zones de shoaling. Ce qui rend la digue beaucoup plus vulnérable aux houles pendant la pleine mer.

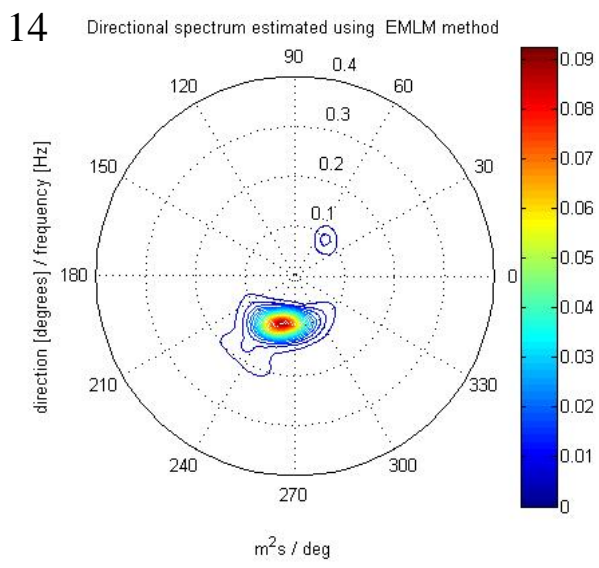

Figure 4. Résultat du spectre "14",

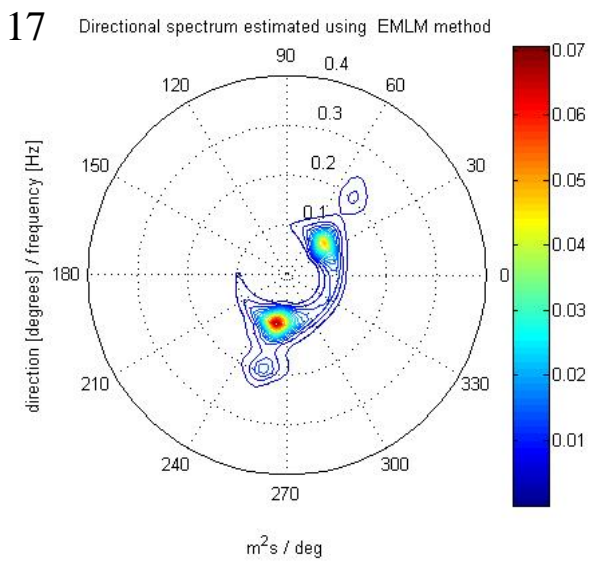

Figure 5. Résultat du spectre "17", 


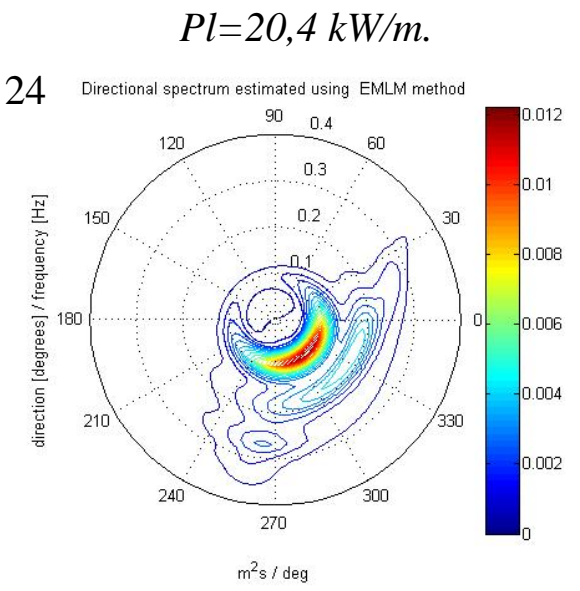

Figure 6. Résultat du spectre "24", $P_{l}=6 \mathrm{~kW} / \mathrm{m}$.

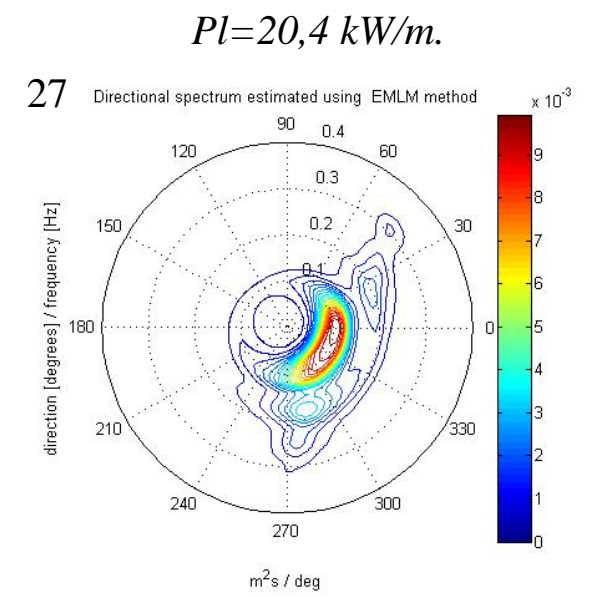

Figure 7. Résultat du spectre "27", $P_{l}=6,7 \mathrm{~kW} / \mathrm{m}$.

\section{Conclusion et perspectives}

L'obliquité des vagues varie de façon significative le long de la digue $\left(180^{\circ} \mathrm{N}\right.$ à $\left.90^{\circ} \mathrm{N}\right)$ d'Esquibien. Les 100 mètres à l'extrémité de la digue sont les plus énergétiques $\left(P_{l}=20 \mathrm{~kW} / \mathrm{m}\right)$. De même, l'île aux vaches est une zone de dissipation majeure $\left(P_{l}=24 \mathrm{~kW} / \mathrm{m}\right.$ au point "33"). En revanche, le haut fond "An Ero" créé une zone de potentiel modéré, selon le niveau d'eau et la hauteur des vagues. Enfin, à l'est de la digue, pour des profondeurs plus importantes, le phénomène de caustique rend le potentiel énergétique plus faible. En définitive, l'étude a permis de caractériser le site d'Esquibien comme un site de fort potentiel en période hivernale. Un des enjeux majeurs à l'implantation d'un récupérateur d'énergie résidera dans sa capacité à résister aux vagues de provenances variées, capables de franchir la digue. Dans ce cadre, le code SWASH pourra constituer un outil en appui au dimensionnement du futur démonstrateur. Pour approfondir le travail présenté, des mesures in-situ devront confirmer les observations qualitatives réalisées. De plus, d'autres simulations pourront être lancées pour couvrir une plus grande diversité d'états de mer à la frontière entrante.

\section{Références bibliographiques}

EPRI (2011). Mapping and assessment of the united states ocean wave energy resource. Technical report, EPRI, Palo Alto.

MICHARD B., DUFOUR G., COSQUER E. (2013). Evaluation préliminaire du potential houlomoteur de 22 sites français en manche et atlantique. Rapport de Recherche IREX R/13/EMA/003 EMACOP, 120 p.

ZIJLEMA M., STELLING G., SMIT P. (2011). Swash: An operational public domain code for simulating wave fields and rapidly varied flows in coastal waters. Coastal Engineering, Vol. 58, pp 992-1012. http://dx.doi.org/10.1016/j.coastaleng.2011.05.015 